Erwerbsarbeitsmobilität in Bremen und Halle/Saale

\title{
Schafft Erwerbsarbeit mehr Verkehr?
}

\section{Die Gestaliung der Arbeitsmärkte hat wesentlichen Einfluß auf Art und Ausmaß der Arbeitsmobilität. Dem Wohnstandort, der Verkehrsmittelwahl und der Gestaltung der Gewerbsarbeit kommen daher besondere Bedeutung zu. An den Beispielen Bremen und Halle/Saale zeigt sich, wie eng diese Fakłoren miłein- ander verknüpft sind und wo Einflußmöglichkeiten liegen.}

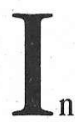

\section{Von Bettina Knothe und Sonja Czyganowski}

n der Alltagsorganisation von Haushalten besitzen die mit der Erwerbsarbeit verbundenen Verkehre eine besondere Bedeutung. Auf der Suche nach einer ökologisch verträglichen Arbeitsmobilität sind vor allem der Wohnstandort der Beschäftigten in Relation zum Betrieb, die Verkehrsmittelwahl im Berufsverkehr und die Potentiale zu einer ökologisch verträglichen Arbeitsmobilität durch Gestaltung der Erwerbsarbeit von Interesse.

\section{Verånderte Arbeitswelt - veränderte Mobilitäit}

Der Blick auf die Rahmenbedingungen der Erwerbsarbeit veranschaulicht eine tiefgreifende Umbruchsituation. Über veränderte individuelle Lebensstile, den Wandel zur Dienstleistungsgesellschaft und neue betriebliche Strukturen werden Mobilitätsnachfrage und Verkehrsorganisa- tion stark verändert. Von zentraler Bedeutung ist hier, daß die allgemein angenommene Normalarbeitszeit kaum noch dem Regelfall des Arbeitsalltags entspricht. Unter Normalarbeitszeit werden die Fünf-Tage-Woche, das freie Wochenende, die 38,5-Stunden-Woche, der sechswöchige Jahresurlaub sowie Schicht-, Abend- und Nacht- oder Wochenendarbeit als Ausnahmefall verstanden. Künftig werden flexible Arbeitszeiten noch stärker zur Realität des Erwerbslebens gehören; für Frauen ist die Flexibilisierung der starren Arbeitszeiten darüber hinaus auch eine Notwendigkeit um an der Erwerbsarbeit teilzunehmen.

Neben den Arbeitszeitstrukturen hängt das Mobilitätsverhalten stark von der Bereitschaft der Menschen ab, weite Arbeitswege in Kauf zu nehmen, um einen Arbeitsplatz zu erhalten oder zu sichern. Lebenszyklische Veränderungen wie Schulabschluß, Aufnahme einer beruflichen Tätigkeit, Haushaltsgründungen und/oder
Zusammenlegungen verstärken die hohen Flexibilitätsanforderungen. Begünstigt wird ein solches Reaktionsschema durch gut ausgebaute Straßen und ein attraktives öffentliches Verkehrsangebot, weil damit die tägliche Überbrückung größerer Entfernungen zwischen Wohn- und Arbeitsort erleichtert wird. Die Bereitschaft zu Pendeln hat sich zudem mit dem knappen Wohnungsmarkt und fehlenden Arbeitsplätzen vor Ort gesteigert. Häufig ist es nur möglich, entweder eine gute Stelle oder eine passende Wohnung in der gleichen Region zu finden. Bindungen der Menschen an Familie, Lebenspartner oder Haus sind weitere Gründe dafür, daß Erwerbstätige lange Anfahrtswege in Betracht ziehen.

\section{Raumbezogene Strategien in Bremen}

In Bremen gibt es zahlreiche betriebliche Aktivitäten, die Mobilität der Erwerbstätigkeit besser $\mathrm{zu}$ organisieren und umweltverträglicher $\mathrm{zu}$ gestalten. Mit dem Begriff des „betrieblichen Mobilitätsmanagement" verbinden sich einerseits gezielte Maßnahmen zur Erhöhung des Anteils von Radverkehr und ÖPNV (z.B. durch Koordination von Arbeits-, Betriebs- und Verkehrszeiten zwischen Betrieb und ÖV-Dienstleister), und andererseits innovative Schritte wie die Bildung von Fahrgemeinschaften, car-pools, etc.

Im Rahmen des Forschungsprojekts ,ökologisch verträgliche Mobilität in Stadtregionen" wïrde am Beispiel Bremen darüber hinaus nach der Einbettung dieser betrieblichen Aktivitäten in die räumliche Planung der Stadt gefragt (1).

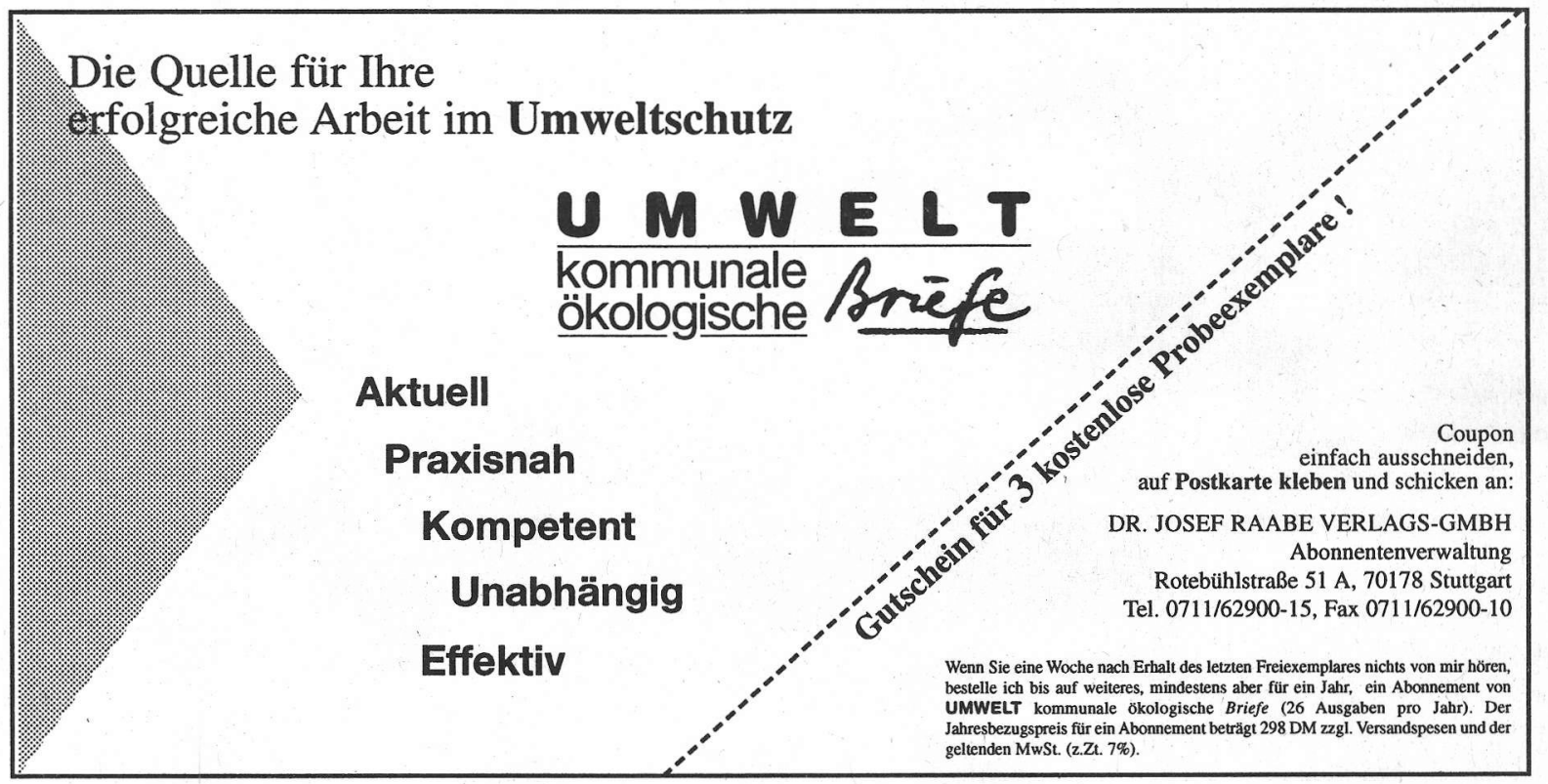


Denn die arbeitsmarktpolitischen Ziele Bremens besitzen auch eine räumliche Komponente: Aus Sicht der Stadtplanung werden vor allem die Innenentwicklung bzw. eine Erneuerung des Flächenpotentials in der Stadt (Flächenrecycling), Funktionsmischung, dezentrale Konzentration neben der Stärkung der Innenstadt und eine ÖPNV-gestützte Siedlungsentwicklung verfolgt (2). Vor dem Hintergrund der stadträumlichen Verteilung und Entwicklung von Wohnstätten und Arbeitsplätzen als auch im Hinblick auf regionale Zusammenhänge (Randwanderung der Beschäftigung, Wettbewerb mit dem Umland) stellt sich die Frage, inwieweit die Gewerbeplanung des Senats über ihre Standortentscheidungen Art, Umfang und ökologische Verträglichkeit der Erwerbsarbeitsmobilität ergänzend zum betrieblichen Mobilitätsmanagement fördern kann (3). Mögliche Ansatzpunkte dazu wären:

- Harmonisierung von Gewerbeplanung und lokalem Arbeitskräfteangebot: Können Arbeitsplätze dort geschaffen oder eventuell dorthin verlagert werden, wo sich die Nachfrage befindet? Oder umgekehrt: Gibt es für Teilräume mit hoher Arbeitslosigkeit Optionen zur gezielten Lokalisierung von Angeboten?

- Integration des Themas „Arbeit“ in die Bauleitplanung: Gibt es differenzierte Kenntnisse über das Arbeitskräfte- und Qualifikationspotential in Teilräumen, mit dem sich aus geplanter Funktionsmischung eine echte, „gelebte“ Mischung ergibt?

\section{- Erwerbsarbeit im Umbruch - Halle/Saale}

In Halle/S. stellt sich die Arbeitsmobilität nicht allein aufgrund der Strukturbrüche in Wirtschaft und Arbeitsmarkt erheblich anders dar als in Bremen. Auch die siedlungsräumliche Ausgangssituation sowie individuelle Einstellungen und Gewohnheiten erfordern eine eigenständige Herangehensweise. Zu Zeiten der DDR sollten die Betriebsstandorte Arbeitsort und Versorgungsort (Gesundheitsversorgung, Einkaufsmöglichkeiten, Kinderbetreuung...) zugleich sein. Feste soziale Bezüge im Umfeld der großen Betriebe waren über einen großen Zeitraum prägend. Häufig waren Wohnund Arbeitsort entweder nicht weit voneinander entfernt (z.B. Werkswohnung) oder durch ein festes ÖPNV-Angebot miteinander verbunden (Halle-Neustadt mit Buna und Leuna). Die Erwerbsarbeit war daher in gewis- ser Weise „ökologisch optimiert“. Längere Anfahrtswege zur Arbeit mußten - auch aus fehlendem Zwang - kaum akzeptiert werden.

Diese Einheit von Arbeiten und Wohnen läßt sich beispielhaft an Halle-Neustadt aufzeigen. Der Bau dieses Stadtteils wurde durch die Ansiedlung großer Chemie-Kombinate Anfang der 60er Jahre in Buna und Leuna induziert. Mit dem Wohnungsangebot sollten Menschen angeworben werden, die gleichzeitig in den Kombinaten Arbeit fanden. In Halle-Neustadt selbst existieren wenig Arbeitsplätze, die vor allem in Dienstleistungsbereichen wie Kinderbetreuung oder Versorgungseinrichtungen angesiedelt sind. Der Schwerpunkt im Hinblick auf Versorgungseinrichtungen liegt dabei auf „wohnortnah“, im Unterschied zu früheren Planungszielen, die Versorgungsschwerpunkte ,betriebsnah“ definiert haben.

Unter den aktuellen Rahmenbedingungen der Arbeitsmarkt- und Erwerbssituation haben diese Strukturen kaum noch Bestand. Die Veränderungen wirken sich tendenziell verkehrserzeugend aus. Dabei spielen vor allem folgende Faktoren eine Rolle:

- Auflösung einer gewachsenen Versorgungsinfrastruktur, die eng mit dem Arbeitsort verbunden war,

- damit einhergehend Auflösung gewachsener ÖPNV-Strukturen (feste Relationen zwischen Arbeits- und Wohnstandorten),

- die Entwicklung neuer dezentralisierter Arbeitsformen wie Heimarbeit,

- eine ausgeprägte Struktur im Bereich des sogenannten zweiten Arbeitsmarktes, die allerdings durch Wegfall der finanziellen Förderung in Frage gestellt ist,

- die nicht vorhandene Bereitschaft der ArbeitnehmerInnen, lange Wege zur Arbeit in Kauf zu nehmen.

Einer der wichtigsten Aspekte in der arbeitspolitischen Diskussion ist die Existenzsicherung von Frauen. Viele Frauen haben nach der Wende eine Umschulung gemacht und trotzdem keinen Arbeitsplatz mehr bekommen, insbesondere Frauen ab 40. Als Ersatz für den verlorenen Arbeitsplatz hat sich folglich eine starke Vereinsstruktur mit der Initiierung zahlreicher Beschäftigungsund Fortbildungsprojekte im zweiten Arbeitsmarkt ausgebildet (Pflege- und Betreuungsbereich, Forschungs- und Beratungsprojekte von und für AkademikerInnen). Berufliche Mobilität wird zunehmend vorausgesetzt und auch massiv staatlich gefördert: So haben viele Frauen in den letzten Jahren mit finanzieller Unterstïtzung des Landes Sachsen-Anhalt einen Führerschein erworben! (Nach der Mobilitätsrichtlinie des Landes gilt dies für alle Fälle, in denen Mobilität vorausgesetzt wird und die Entfernung eine bestimmte Distanz überschreitet).

Eine ökologisch verträgliche Mobilitätsgestaltung ist also nicht nur vor schwerwiegende konkurrierende Güterabwägungen gestellt, nach denen die Förderung der Erwerbsarbeit viel höher gewichtet wird als die Frage ,Verkehrserzeugung oder einsparung, sondern findet teilweise auch direkt kontraproduktive Maßnahmen der Politik vor. In diesem Kontext könnte ein bescheidener Beitrag zur (ökologischen) Optimierung der Arbeitsmobilität darin bestehen, laufende Aktivitäten im zweiten. Arbeitsmarkt (Qualifizierung, Weiterbildung) auf ihre sprichwörtlich verkehrsgünstigste Organisationsform hin zu überprïfen. Das gesellschaftliche Problembewußtsein hierfür ist allerdings begrenzt: In einem Umfeld weitgehender Deindustrialisierung ist nicht die Arbeitsmobilität, sondern die fehlende Erwerbsarbeit das zentrale Problem.

\section{Anmerkungen}

1) Zentrale wirtschafts- und strukturpolitische Ziele bremischer Stadtpolitik liegen u.a. in der Sicherung und Schaffung attraktiver Arbeitsplätze, in der Schaffung von Beschäftigungsmöglichkeiten für Frauen, einer engeren Verzahnung von arbeitsmarktpolitischen mit strukturpolitischen Maßnahmen und in der Verbesserung der Qualifikationsstruktur. - Niedersächsisches Landesministerium und Senator für Umweltschutz und Stadtentwicklung der Freien Hansestadt Bremen: Konzept für eine regionale Infrastrukturpolitik im Raum der gemeinsamen Landesplanung Bremen/Niedersachsen (Strukturkonzeption Bremen/Niedersachsen 2010). 1994

2) S. Herlyn, Abteilung Raumordnung und Stadtentwicklung beim Senator für Bau, Verkehr und Stadtentwicklung: Perspektivenwechsel? - Die Diskussion über die Bremer Stadtentwicklung nach der Veränderung der politischen Konstellation; Workshop "Stadtentwicklung in Bremen Perspektiven für eine ökologisch verträglichere Mobilität"; Vortrag vom 19.4.1996

3) Zwischenbericht: Ökologisch verträgliche Mobilität in Stadtregionen, Projektbereich D: Personenverkehr, Teilprojekt 12: Arbeitsalltragsmobilität/Erwerbsarbeitsmobilität; April 1996

\section{Die Autorinnen}

Bettina Knothe, Dipl.-Biol., und Sonja Czyganowski stud.-oec, sind beide Mitarbeiterinnen des IÖW, Regionalbüro NRW, Völkinger Str. 9 , 42285 Wuppertal, Tel. (02 02) 80530 
(c) 20I0 Authors; licensee IÖW and oekom verlag. This is an article distributed under the terms of the Creative Commons Attribution Non-Commercial No Derivates License (http://creativecommons.org/licenses/by-nc-nd/3.o/), which permits unrestricted use, distribution, and reproduction in any medium, provided the original work is properly cited. 